\title{
Comparison of serum anti-neuronal antibody levels in patients having autism spectrum disorder with and without regression
}

\author{
Cihan Aslan ${ }^{1 \oplus}$, Bahadır Konuşkan ${ }^{2 \oplus}$, Burçin Şener ${ }^{3 \oplus}$, Fatih Ünal ${ }^{1 \oplus}$ \\ Departments of ${ }^{1}$ Child and Adolescent Psychiatry, ${ }^{2}$ Pediatric Neurology and ${ }^{3}$ Medical Microbiology, Hacettepe University Faculty of \\ Medicine, Ankara, Turkey.
}

\begin{abstract}
Background. Autism Spectrum Disorders (ASD) may present with a delay in social and communication development, or less frequently, with regression in social and language skills. The reasons for this difference in clinical presentation are unknown, and the regressive symptoms in the second group suggest an acquired process.

Methods. We investigated serum autoantibodies in these two types of ASD in a cross-sectional design in a total of 50 children, 24 with autistic regression and 26 with classical ASD according to the DSM-5 criteria. Clinical assessment by the Childhood Autism Rating Scale (CARS) and Ankara Developmental Screening Test (ADST), parental questionnaires consisting of the Aberrant Behavior Checklist (ABC) and Autism Behavior Checklist $(\mathrm{AuBC})$ were completed. Serum samples were tested for anti-neuronal antibodies including anti-N-methyl-Daspartate receptor (NMDAR), anti-contactin-associated protein (CASPR2), anti-leucine rich glioma inactivated 1 (LG1), anti-glutamate type 2-amino-3-propionic Acid (AMPA) 1-2, anti-gamma amino butyric acid (GABA) B, anti-dipeptidyl aminopeptidase-like protein 6 (DPPX) and anti-glutamic acid decarboxylase 65(GAD).
\end{abstract}

Results. Serum anti-GAD antibodies were at detectable levels in five $(20.8 \%)$ patients with autistic regression, of whom three had 2 to 4 -fold increased titers, and in none of the patients with classical ASD. The age of the father at the patient's birth and the duration of autistic regression correlated with anti-GAD IgG levels (P: 0,045, P: 0.855 respectively) in the ASD-regression group. No other antibodies were detected in either group.

Conclusions. Our results do not suggest a causative role of anti-neuronal antibodies, but the possibility of an autoimmune process accompanying regressive symptoms in a small subgroup of ASD.

Key words: autism, regression, autoimmunity, antibody mediated, encephalitis.

Autism spectrum disorder (ASD) is a neurodevelopmental condition involving deficiencies in interaction, communication, and social interest. Its etiopathogenesis, phenotype and course are heterogeneous. About one third of ASD cases show sudden or gradual loss of previously attained skills at 12-24 months of

¿ Cihan Aslan

cihanaslan@yahoo.com

Received 12th November 2020, revised 31st January 2021, accepted 23rd February 2021.

10th International Congress on Psychopharmacology \& 6th International Symposium on Child and Adolescent Psychopharmacology, April 25-29, 2018, Antalya, Turkey. age. ${ }^{1}$ Some authors consider regression as a subtype of ASD with a particular etiology and course and also poorer outcome, while others suggest classical and regressive ASD cases have similar behavioral and adaptive outcomes. ${ }^{2}$

ASD can be associated with immune dysfunction, as supported by studies on cell profiles of the immune system, plasma cytokine levels, serum immunoglobulins, and autoantibodies against neuronal proteins. ${ }^{3}$ The various targets of the antibodies detected in ASD cases include multiple brain regions and somatic tissues: neuronal membranes, folate receptors, mitochondria, gastrointestinal epithelial cells, 
endothelial cells, ribosome $\mathrm{P}$, or antinuclear antibody., The heterogeneity of targets and lack of consistency among results of the studies warrant further specific studies in order to clarify the role of antibodies in ASD. Uncertainty exists about the autoimmune process involving a causative relationship or an immune activation in response to an unknown etiological agent. As ASD is a broad and heterogeneous condition, the role of inflammatory pathways may be more prominent in certain subgroups, such as ASD with regressive course. Febrile diseases, family history of autoimmune disorders, elevated serum inflammatory biomarkers have been observed more frequently in this group..$^{5-7}$ HLA polymorphisms detected in autoimmune disorders were also described in ASD with regression. ${ }^{8}$ Microglial activation leading to neuronal and synaptic loss was shown in the central nervous system (CNS) in up to 69\% of ASD cases, mostly in those with regressive course. ${ }^{9}$ Recently auto-antibodies against CNS antigens have been implicated in the etiology of ASD; a systematic review underlined the need for studies on autoimmune encephalitis and HLA haplotypes. ${ }^{4}$ Indeed cases and experimental animal models of autoimmune encephalitis can exhibit autistic behavior. ${ }^{10,11}$ Another clinical example is the Landau-Kleffner Syndrome, a steroid-responsive epileptic syndrome with autoimmune or inflammatory pathogenesis and autistic features. ${ }^{12}$

Regression remains an intriguing feature adding to the clinical variability of ASD. However, the etiology and pathogenesis underlying this particular clinical characteristic are unclear. In this study, we investigated serum autoantibodies in ASD with and without regression.

\section{Material and Methods}

This cross-sectional case-control study was performed in Hacettepe University Faculty of Medicine following the approval of Hacettepe University Non-Interventional Clinical Studies Ethics Committee (\#GO 16/73-09).

\section{Patient Groups}

Children 2-6 years old followed-up in the Child and Adolescent Psychiatry Department for ASD based on DSM-5 diagnostic criteria were prospectively recruited after parental consent. Patientsdiagnosed withchildhood disintegrative disorder, children with regression starting after age 36 months, those who lost motor skills or toilet training, those with associated epilepsy or electroencephalogram (EEG) abnormality were not included. Subjects with hearing loss, genetic or metabolic syndromes, and any concomitant immunotherapy were excluded from the study.

\section{Definition of Regression}

Symptoms of regression were assessed through semi-structured interviews with parents, from hospital records and, whenever available, video recordings. Acquisition of early developmental milestones and criteria for regression were identified as defined and used in the literature ${ }^{13}$ :

- Significant loss in communication skills (loss of at least 5 words in a child who could speak earlier, or loss of mimics and / or ability to understand words, sentences or interpretations in a child whose maximum vocabulary was 4 meaningful words when the regression started) before 36 months of age for more than 3 months, and

- Significant loss in social skills (ASD related symptoms in at least $1 / 3$ of social skills or behavioral patterns defined as social smile, eye contact, joint attention, responding to name, waving hand, pointing, interest in playing with others, imaginary play, functional use of objects and toys, repetitive / stereotypic behavior or limited interests) before 36 months of age for more than 3 months.

Regression was identified as acute when significant loss occurred over 4-6 weeks and gradual (or subacute/chronic) when it occurred over $>6$ weeks. Physical factors (high fever, infection, seizure, trauma, vaccination etc.) and environmental changes associated with onset 
of regression such as birth of a sibling, loss of a family member, moving, change of caregiver, decreased quality of stimuli were recorded.

\section{Psychiatric Evaluation}

Psychiatric evaluation included the Childhood Autism Rating Scale (CARS) to confirm the diagnosis and assess disease severity and Ankara Developmental Screening Test (ADST) for global development. Parents were asked to fill the Autism Behavior Checklist (AuBC) and the Aberrant Behavior Checklist (ABC). ${ }^{14-}$ 17 Sociodemographic and clinical information was recorded by a standard form prepared for this study. Medical problems of the patient and family members such as recurrent infections, asthma/allergies, sleep problems, recurrent constipation/diarrhea, psychiatric symptoms or neurodevelopmental delay were recorded.

\section{Measuring Serum Antibodies}

Custom-made panel of antibodies have been designed based on literature data: anti-Nmethyl-D-aspartate receptor (NMDAR), anti-contactin-associated protein (CASPR2), anti-leucine rich glioma inactivated 1 (LG1), anti-glutamate type 2-amino-3-propionic Acid (AMPA) 1-2, anti-gamma amino butyric acid (GABA) B, anti-dipeptidyl aminopeptidase-like protein 6 (DPPX) were tested in serum samples stored at $-80^{\circ} \mathrm{C}$. An indirect immunofluorescent antibody assay on transfected cells (Mosaic 6, Euroimmun, Perkin Elmer, Lubeck, Germany) was applied in accordance with the manufacturer's recommendations. ${ }^{18}$ Antibodies against glutamic acid decarboxylase isoform 65 (anti-GAD $65 \mathrm{IgG}$ ) were tested using the radioimmune assay (RIA) method (Beckman Coulter, Czech Republic). ${ }^{19}$ Positive controls for the investigated autoantibodies were included in the test kits used in the study.

\section{Statistical Analysis}

Quantitative data was assessed for normal distribution using Kolmogorov-Smirnov test. Comparisons of numeric data between 2 groups was done with independent sample t-test for variables with normal distribution, and Mann Whitney-U test for those with non-normal distribution. Categorical (qualitative) variables were expressed as number and percentage. Pearson Chi-Square and Fisher's Exact ChiSquare tests were used depending on the status of the expected frequencies. The power of the correlation between variables was evaluated by Pearson or Spearman Rho correlation coefficients. All analyses were performed through the Statistical Program for Social Sciences (SPSS 21.0) in a two-tailed pattern with $<0.05$ as margin of limit for statistical significance.

\section{Results}

\section{Demographic and Clinical Characteristics}

The ASD series $(n=50)$ comprised two groups based on the presence of a history of regression: the "regression" $(\mathrm{n}=24)$ and "classical course" $(n=26)$ groups. The two groups were similar in distribution of mean age $(50.7 \pm 13.3$ and $50.1 \pm 12.0$ months respectively) and gender (79.2\% male, $20.8 \%$ female and $84.6 \%$ male, $15.4 \%$ female) as well as the prevalence of accompanying medical conditions in patients and their first- and second-degree relatives. None had any systemic disorders or chronic medication, and all patients had been treated with behavioral interventions only.

Early developmental milestones: language, toilet training, walking had been acquired earlier in the regression group (Table I). The two groups showed similar CARS, ADST, AuBC and ABC scores $(\mathrm{t}=0.35 / \mathrm{p}>0.05, \mathrm{t}=-1.4 / \mathrm{p}>0.05, \mathrm{t}=$ $1.1 / \mathrm{p}>0.05, \mathrm{t}=1.6 / \mathrm{p}>0.05)$.

\section{Clinical Characteristics of the Regression Group}

The mean age of onset of regression was $24 \pm 8.6$ (13-36) months; blood samples were collected at mean $50.7 \pm 13.3$ months, the interval between onset of symptoms and blood sampling was 26.7 \pm 11.6 (6-42) months. The pattern of regression 
Table I. Clinical data.

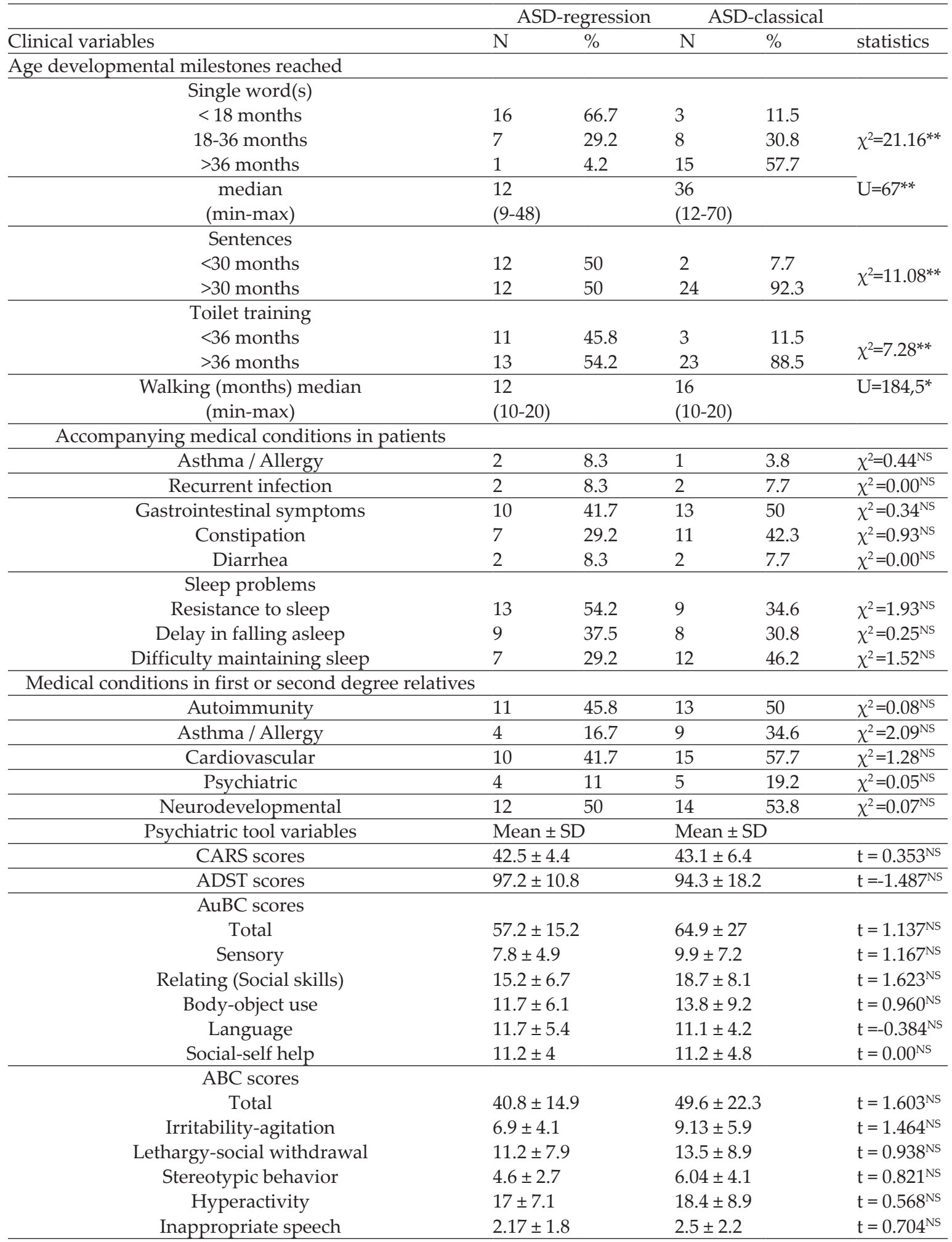

n: number, U: Mann Whitney U test, min: minimum, max: maximum, $\chi^{2}$ : chi square test, $\mathrm{t}$ test, SD: standard deviation, NS: not significant, ASD: autism spectrum disorder, CARS: childhood autism rating scale, ADST: Ankara Developmental screening test, $A u B C$ : autism behavior checklist, $A B C$ : aberrant behavior checklist, ${ }^{*} \mathrm{p}<0.05,{ }^{* *} \mathrm{p}<0.01$ 
documented: acute loss of language and social skills ( $\mathrm{n}=4$ children, $16.7 \%$ ), gradual loss of language and social skills $(n=13,54.2 \%)$, gradual loss of language skills ( $n=3,12.4 \%)$ and gradual loss in language but sudden loss in social skills $(n=4,16.7 \%)$. Regression was associated with a physical illness or condition including febrile illness, febrile convulsion, vaccination, or trauma in $12(50 \%)$ and a change in the child's environment in 7 (29\%) children respectively. Similar rates were reported in the "classical course" group: physical illness/disturbance in 11 patients $(42 \%)$, environmental changes in 8 patients $(30 \%)$ were recognized at the time of the first symptoms. These associations were verified by parental reports.

The younger the child made sentences and the more words he/she pronounced before regression, the more words he regained after intervention ('spearman correlation coefficient' P: -0.559 , p: 0.005 and P: 0.421, p: 0.041). Cases with gradual loss in social skills had higher mean scores in AuBC total $(62.8 \pm 14.1$ vs. 47.5 $\pm 12.4, \mathrm{t}=-2.5 / \mathrm{p}<0.05)$ and AuBC social-self-help subscales $(8.5 \pm 2.6$ vs. $12.6 \pm 4, \mathrm{t}=-2.6 / \mathrm{p}<0.05)$, but fewer words before regression (mean 42.5, range $5-300$ vs. mean 10 , range $0-400$ words, $U=$ $27 / \mathrm{p}<0.05)$ compared to those with sudden loss.

\section{Autoantibodies in Regression and Classical Course Groups}

Anti-NMDAR, anti-AMPA 1-2, anti-CASPR2, anti-LGI1, anti-GABAB, anti-DPPX antibodies were not detected in either group. The detection range of anti-GAD titers was 0.09$4.33 \mathrm{U} / \mathrm{ml}$. Titers above the upper limit ( $1 \mathrm{U} /$ $\mathrm{mL})$ were measured in five $(20.8 \%)$ patients of the regression group and none of the "classical course" group $\left(\chi^{2}=6.019, \mathrm{p}<0.05\right)$. Regressive cases positive and negative for anti-GAD antibody were the same in terms of sociodemographic characteristics, early developmental milestones, medical and family history, test results, or interval between onset of symptoms and collection of the serum sample (Table II).
Three patients whose anti-GAD titer was more than twice the normal titer included two boys and a girl with developmental arrest predominantly in the language area. The highest titer, $4.33 \mathrm{u} /$ $\mathrm{mL}$, was documented in a 5-year-old girl having an acute language arrest followed by regression over 2 years after an upper respiratory infection. She had 15-20 words before regression and had regained 50-60 words at the time of the study. A 5-year-old boy with anti-GAD titer of $3.01 \mathrm{u} /$ $\mathrm{mL}$, a family history of developmental problems and ASD in distant relatives demonstrated subacute arrest and subsequent regression after familial and environmental changes at age 2 years. The third patient, aged 3,5 years and anti-GAD titer $2.18 \mathrm{u} / \mathrm{mL}$ at the time of study, had a family history of autoimmune disease in grandparents and schizophrenia in maternal uncle. He showed acute language arrest at age 1.5 years after otitis and the birth of a sibling, followed by subacute regression. His hearing test was normal. Currently they have been under therapy and follow-up. Three of the anti-GAD positive patients were re-evaluated after the study and screened for autoimmune diseases: no significant clinical or laboratory findings were detected.

Correlation analysis between the clinical scores and anti-GAD autoantibody levels of those five patients using Spearman test showed no correlation. Analysis within the entire ASDregression group showed the age of father at birth and the duration of regression correlated positively with the level of anti-GAD antibody (pearson correlation, P: 0,045 p: 0,025 and P:0,855 p:0.00)

\section{Discussion}

This study aimed to compare ASD with and without regression according to clinical and certain laboratory features. Demographics, medical history, developmental characteristics, clinical and environmental variables were not found to differ significantly between these two groups. Early developmental milestones (i.e., language skills, toilet training, walking) 
Table II. Clinical characteristics of the regression group.

\begin{tabular}{|c|c|c|}
\hline & $\begin{array}{l}\text { Anti-GAD-positive } \\
(\mathrm{n}=5,20 \%)\end{array}$ & $\begin{array}{l}\text { Anti-GAD-negative } \\
(\mathrm{n}=19,80 \%)\end{array}$ \\
\hline Age range at onset (months) & $18-25$ & $13-36$ \\
\hline Age range at study (years) & $3.5-5$ & $2-6$ \\
\hline Gender $(\mathrm{M} / \mathrm{F})$ & $4 / 1$ & $16 / 3$ \\
\hline $\begin{array}{l}\text { Time (months) between onset of symptoms-collection of sample, } \\
\text { mean } \pm \mathrm{SD}^{*}\end{array}$ & $33.4 \pm 7.6$ & $25 \pm 12$ \\
\hline CARS scores, mean \pm SD $^{*}$ & $41.8 \pm 4.1$ & $42.7 \pm 4.6$ \\
\hline ADST scores, mean \pm SD $^{*}$ & $98.6 \pm 13$ & $96.9 \pm 9$ \\
\hline \multicolumn{3}{|l|}{ AuBC scores, mean $\pm \mathrm{SD}^{*}$} \\
\hline Total & $54.8 \pm 15.3$ & $58.4 \pm 15.5$ \\
\hline Sensory & $7.2 \pm 5.6$ & $8 \pm 4.8$ \\
\hline Relating (Social skills) & $15.8 \pm 11$ & $15 \pm 5.3$ \\
\hline Body-object use & $10 \pm 3.8$ & $12.1 \pm 6.6$ \\
\hline Language & $11.4 \pm 3.9$ & $11.7 \pm 5.8$ \\
\hline Social-self help & $10.4 \pm 3$ & $11.4 \pm 4.3$ \\
\hline \multicolumn{3}{|l|}{ ABC scores, mean $\pm \mathrm{SD}^{*}$} \\
\hline Total & $36.6 \pm 9.3$ & $42 \pm 16.1$ \\
\hline Irritability-agitation & $6.4 \pm 4.3$ & $7.1 \pm 4.2$ \\
\hline Lethargy-social withdrawal & $10 \pm 8.9$ & $11.5 \pm 7.8$ \\
\hline Stereotypic behavior & $1.8 \pm 2.1$ & $3.8 \pm 2.7$ \\
\hline Hyperactivity & $16.4 \pm 4$ & $17.2 \pm 7.8$ \\
\hline Inappropriate speech & $2 \pm 2.9$ & $2.2 \pm 1.6$ \\
\hline \multicolumn{3}{|l|}{ Onset patterns: loss of skills } \\
\hline Sudden social \& Language & $1(20 \%)$ & $3(15 \%)$ \\
\hline Gradual social \& Language & $3(60 \%)$ & $10(50 \%)$ \\
\hline Sudden social \& Gradual Language & - & $4(21 \%)$ \\
\hline Gradual social \& Sudden Language & $1(20 \%)$ & $2(10 \%)$ \\
\hline
\end{tabular}

n: number, SD: standard deviation, M: male, F: female, Anti-GAD: anti-glutamic acid decarboxylase, CARS: childhood autism rating scale, ADST: Ankara Developmental screening test, AuBC: autism behavior checklist, ABC: aberrant behavior checklist, ${ }^{*} \mathrm{p}<0.05$, t test, not significant

were attained earlier in the regressive group. In fact, some of the ASD-regression cases had a developmental pattern very similar to typically developing children. Overall, these clinical findings were similar to those in the literature. ${ }^{8}$

The immune system has been implicated in the etiopathogenesis of ASD although it is unclear whether this is a causative relationship or an indirect association. The hypothesis of immune dysfunctionmightbemorerelevanttoaparticular subgroup within ASD, namely, the group ASD-regression in this study. Autoimmunity, neuroinflammation, and microglial activity resulting in neuronal death and synaptic loss have been considered in the pathogenesis of autistic regression. An association of ASD with autoimmune and allergic diseases has been shown in a large nationwide cohort. ${ }^{20}$ The frequency of febrile illnesses in the 6 months prior to initial manifestations of ASD, and the higher prevalence of autoimmune disorders including type 1 diabetes and thyroiditis in the family also may point towards autoimmune pathogenesis in ASD with regression. ${ }^{21}$ Recent literature observed developmental milestones being reached significantly earlier in ASD cases with regression than in classical ASD: this 
may also imply the role of an acquired process compatible with autoimmunity. ${ }^{22}$ Rather than being the primary cause, the immune response might act as a "biological hit" in a child with genetic or metabolic predisposition to ASD.$^{23}$ For this reason we studied antineuronal antibodies described in the literature as associated with behavioral and autistic symptoms, and became part of the diagnostic work-up of acute or subacute encephalopathies and epilepsies. ${ }^{24}$ The commercial kits include antineuronal antibodies demonstrated in previous laboratory and clinical studies on autoimmune CNS disorders as well as in the limited number of prior studies in autism. The most common anti-neuronal antibody found in children is anti-NMDAR. It causes an encephalitis manifesting with behavioral changes, loss of social, speech, and communication skills, all symptoms evocative of the autistic regression in young children. ${ }^{25}$ In fact, two children demonstrating autistic regression when 2 years old were reported with anti-NMDAR antibody positivity in their serum and cerebrospinal fluid (CSF). ${ }^{26}$ Neuronal AMPA receptors, GABA $B$ receptors and VGKC complex have a role in ASD as shown in laboratory and clinical studies. ${ }^{27-29}$ The absence of these antibodies in our series argues against these receptors being affected by autoimmunity in young children with ASD. Alternatively, by testing the serum but not the CSF and antibodies of the IgG type only, we may have overlooked some antibodies: serum can be negative in as many as $14 \%$ of anti-NMDAR encephalitis cases, and certain anti-NMDAR antibodies are of the $\operatorname{Ig} \mathrm{A}$ and $\operatorname{IgM}$ types, undetected in routine assays. Also, antibody titers can decrease spontaneously within months regardless of the clinical course. ${ }^{30}$ Serum samples can be useful in antibody-mediated CNS disorders where the blood-brain barrier is impaired, but this is not the case in the majority of childhood autism cases.

The only antibodies found in our series were anti-GAD65 IgG. These are detected in diabetes but also in various autoimmune conditions. The prevalence of anti-GAD positivity in healthy children is consistently reported at around $2 \% .{ }^{31}$ Neurological phenotypes associated with antiGAD antibody are the stiff person syndrome, temporal lobe epilepsy, cerebellar ataxia and limbic encephalitis. ${ }^{32}$ These antibodies affect cerebellar Purkinje cells which have been implied in the pathogenesis of ASD: for instance, maternal anti-GAD antibodies may cause loss of fetal Purkinje cells. ${ }^{33}$ AntiGAD seropositivity at low titers in our study may suggest a nonspecific response against undefined, possibly neural antigenic targets rather than a direct etiological finding. ${ }^{34}$ Followup of the titers may be envisaged in future studies in order to support or refute an ongoing secondary immune response. In our study, serum was tested: although the concordance rate of CSF and serum samples is high in antiGAD antibody-related neurological syndromes, fluctuations in anti-GAD65 levels and false negativity are possible and CSF analysis might be more specific, as some anti-GAD65 antibody is synthesized intrathecally. ${ }^{35-37}$ The GAD enzyme has two isoforms, GAD 65 and 67 , which are both reduced in the cerebellum in autism. ${ }^{38}$ Our tests did not comprise antiGAD67 antibodies; although they are rare in children, some cases might have been missed for this reason. ${ }^{38}$ Another possible factor is the interval between our testing for antibodies and the onset of symptoms. This may constitute more of a drawback for other antineuronal antibodies, as anti-GAD titers tend to persist even after treatment. ${ }^{39}$ Among the few previous studies on ASD and anti-GAD antibody, one showed $3 / 20(15 \%)$ were positive while others reported all samples as seronegative. ${ }^{36,40-42}$ These studies did not include clinical assessment. To the best of our knowledge, this is the first report investigating anti-GAD in ASD comparing classical and regressive patterns.

The question of immunomodulatory medication arises especially in the current era of personalized treatments. Successful outcomes obtained with steroids in the LandauKleffner Syndrome and in autism secondary to Autoimmune Lymphoproliferative Syndrome 
(ALPS) suggest their use in the treatment of regressive symptoms of autism. ${ }^{43,44}$ In regressive ASD, a recent retrospective study reported more clinical improvement in steroidtreated than non-steroid groups and a 12-week, randomized, single-blinded, placebo controlled trial showed a significant effect in CARS and $\mathrm{ABC}$. ${ }^{45,46}$ Intravenous immunoglobulin treatment evaluated in an open-label study demonstrated some improvement in $A B C$ and/or Social Responsiveness Scale (SRS) in the majority of children of children with autoimmune encephalitis and ASD. ${ }^{47} \mathrm{~A}$ recent review concluded that some subsets of ASD where immune-mediated mechanisms play a role may benefit from immunomodulatory treatment. ${ }^{48}$

Immune mechanisms' role in ASD has been subjected to numerous studies in the last decade; however, as it is not possible to examine the immune system comprehensively in a single study, hypotheses drawn from observational reports or published cases need to be investigated longitudinally in a multicentric fashion. Our results comparing two subtypes of autism can only be attributed to a certain group of patients. On the other hand, the strengths of this study include the comparability of groups in terms of symptom severity, developmental level and age range: these reduce any possible bias in the reporting of regression. The single center design allows homogeneity of patient groups and methodology. The main limitations of the study are its cross-sectional rather than longitudinal design, small sample size precluding generalization of our findings, and variations in the interval between the onset of regression and sample collection. Some information obtained from caregivers might introduce a recall bias, as under-reporting of the age when regression started is common. ${ }^{49} \mathrm{On}$ the other hand, parents of older children tend to report that regression begun later. This effect is described as "telescoping effect" and confirmed with a recent meta-analysis. ${ }^{1}$ To alleviate this, we applied strict definitions of early developmental stages, symptoms, duration, triggering factors at onset, losses and regains in language. Whereas retrospective design is an inevitable limitation, prospective studies have their own limitations in distinguishing loss of skills from developmental delay. ${ }^{50}$ In our study, antibodies were not tested in CSF which is more sensitive for most antibodies, but limited by ethical issues. Testing different age groups, re-testing after clinical remission and clinicallaboratory correlation could contribute to such studies because neurological manifestations associated with an antibody can vary depending on the developmental stage and age-dependent vulnerability of the nervous system. Thus, future studies should adopt longitudinal observation of children at risk according to the age of onset and using standard assessment methods.

\section{Acknowledgments}

This study was supported by the Hacettepe University Scientific Research Projects Coordination Unit (project no: THD-201610593).

\section{Author contribution}

The authors confirm contribution to the paper as follows: study conception and design: CA, $\mathrm{BK}, \mathrm{FÜ}$; data collection: CA, BK, FÜ; analysis and interpretation of results: $\mathrm{CA}, \mathrm{BS}$. FÜ; draft manuscript preparation: CA, BK, BŞ, FÜ. All authors reviewed the results and approved the final version of the manuscript.

\section{Ethical approval}

This cross-sectional case-control study was performed in Hacettepe University Faculty of Medicine following the approval of Hacettepe University Non-Interventional Clinical Studies Ethics Committee (\#GO 16/73-09).

\section{Conflict of interest}

The authors declare that they have no conflicts of interest. 


\section{REFERENCES}

1. Barger BD, Campbell JM, McDonough JD. Prevalence and onset of regression within autism spectrum disorders: a meta-analytic review. J Autism Dev Disord 2013; 43: 817-828.

2. Jones LA, Campbell JM. Clinical characteristics associated with language regression for children with autism spectrum disorders. J Autism Dev Disord 2010; 40: 54-62.

3. Hughes HK, Mills Ko E, Rose D, Ashwood P. Immune dysfunction and autoimmunity as pathological mechanisms in autism spectrum disorders. Front Cell Neurosci 2018; 12: 405.

4. Zou T, Liu J, Zhang X, Tang H, Song Y, Kong X. Autoantibody and autism spectrum disorder: a systematic review. Res Autism Spectr Disord 2020; 75: 101568.

5. Saghazadeh A, Ataeinia B, Keynejad K, Abdolalizadeh A, Hirbod-Mobarakeh A, Rezaei N. A meta-analysis of pro-inflammatory cytokines in autism spectrum disorders: effects of age, gender, and latitude. J Psychiatr Res 2019; 115: 90-102.

6. Prosperi M, Guiducci L, Peroni DG, et al. Inflammatory biomarkers are correlated with some forms of regressive autism spectrum disorder. Brain Sci 2019; 9: 366.

7. Han YM, Chan AS, Sze SL, et al. Altered immune function associated with disordered neural connectivity and executive dysfunctions: a neurophysiological study on children with autism spectrum disorders. Res Autism Spectr Disord 2013; 7: 662-674.

8. Boterberg S, Charman T, Marschik PB, Bölte S, Roeyers $\mathrm{H}$. Regression in autism spectrum disorder: a critical overview of retrospective findings and recommendations for future research. Neurosci Biobehav Rev 2019; 102: 24-55.

9. Morgan JT, Chana G, Pardo CA, et al. Microglial activation and increased microglial density observed in the dorsolateral prefrontal cortex in autism. Biol Psychiatry 2010; 68: 368-376.

10. Won H, Lee HR, Gee HY, et al. Autistic-like social behaviour in Shank2-mutant mice improved by restoring NMDA receptor function. Nature 2012; 486: $261-265$.

11. Hacohen Y, Wright S, Gadian J, et al. N-methyl-daspartate (NMDA) receptor antibodies encephalitis mimicking an autistic regression. Dev Med Child Neurol 2016; 58: 1092-1094.

12. Caraballo RH, Cejas N, Chamorro N, Kaltenmeier MC, Fortini S, Soprano AM. Landau-Kleffner syndrome: a study of 29 patients. Seizure 2014; 23: 98-104.
13. Ozonoff S, Williams BJ, Landa R. Parental report of the early development of children with regressive autism: the delays-plus-regression phenotype. Autism 2005; 9: 461-486.

14. Schopler E, Reichler RJ, DeVellis RF, Daly K. Toward objective classification of childhood autism: Childhood Autism Rating Scale (CARS). J Autism Dev Disord 1980; 10: 91-103.

15. Savasır I, Sezgin N, Erol N. Ankara Gelişim Tarama Envanteri. (3. Basım) Ankara: Türk Psikologlar Derneği Yayınları, 1998.

16. Krug DA, Arick J, Almond P. Behavior checklist for identifying severely handicapped individuals with high levels of autistic behavior. J Child Psychol Psychiatry 1980; 21: 221-229.

17. Aman MG, Singh NN, Stewart AW, Field CJ. The aberrant behavior checklist: a behavior rating scale for the assessment of treatment effects. Am J Ment Defic 1985; 89: 485-491.

18. Ricken G, Schwaiger C, De Simoni D, et al Detection methods for autoantibodies in suspected autoimmune encephalitis. Front Neurol 2018; 9: 841.

19. Malter MP, Helmstaedter C, Urbach H, Vincent A, Bien CG. Antibodies to glutamic acid decarboxylase define a form of limbic encephalitis. Ann Neurol 2010; 67: 470-478.

20. Chen MH, Su TP, Chen YS, et al. Comorbidity of allergic and autoimmune diseases in patients with autism spectrum disorder: a nationwide populationbased study. Res Autism Spectr Disord 2013; 7: 205212.

21. Scott O, Shi D, Andriashek D, Clark B, Goez HR. Clinical clues for autoimmunity and neuroinflammation in patients with autistic regression. Dev Med Child Neurol 2017; 59: 947-951.

22. Ozonoff S, Iosif AM, Young GS, et al. Onset patterns in autism: correspondence between home video and parent report. J Am Acad Child Adolesc Psychiatry 2011; 50: 796-806.e1.

23. Young AM, Chakrabarti B, Roberts D, Lai MC, Suckling J, Baron-Cohen S. From molecules to neural morphology: understanding neuroinflammation in autism spectrum condition. Mol Autism 2016; 7: 9.

24. Brenton JN, Goodkin HP. Antibody-mediated autoimmune encephalitis in childhood. Pediatr Neurol 2016; 60: 13-23.

25. Scott O, Richer L, Forbes K, et al. Anti-N-methyl-Daspartate (NMDA) receptor encephalitis: an unusual cause of autistic regression in a toddler. J Child Neurol 2014; 29: 691-694.

26. Hacohen Y, Wright S, Gadian J, et al. N-methyl-daspartate (NMDA) receptor antibodies encephalitis mimicking an autistic regression. Dev Med Child Neurol 2016; 58: 1092-1094. 
27. Peñagarikano O, Geschwind DH. What does CNTNAP2 reveal about autism spectrum disorder? Trends Mol Med 2012; 18: 156-163.

28. Oblak AL, Gibbs TT, Blatt GJ. Decreased GABA(B) receptors in the cingulate cortex and fusiform gyrus in autism. J Neurochem 2010; 114: 1414-1423.

29. O'Connor EC, Bariselli S, Bellone C. Synaptic basis of social dysfunction: a focus on postsynaptic proteins linking group-I mGluRs with AMPARs and NMDARs. Eur J Neurosci 2014; 39: 1114-1129.

30. Gresa-Arribas N, Titulaer MJ, Torrents A, et al. Antibody titres at diagnosis and during follow-up of anti-NMDA receptor encephalitis: a retrospective study. Lancet Neurol 2014; 13: 167-177.

31. Cambuli VM, Incani M, Cossu E, et al. Prevalence of type 1 diabetes autoantibodies (GADA, IA2, and IAA) in overweight and obese children. Diabetes Care 2010; 33: 820-822.

32. Menon D, Menon RN, Kumar $\mathrm{H}$, et al. The expanding spectrum of pediatric anti-glutamic acid decarboxylase antibody mediated CNS disease-a chance association? Neuroimmunol Neuroinflamm 2016; 3: 219-224.

33. Rout UK, Dhossche DM. A pathogenetic model of autism involving Purkinje cell loss through antiGAD antibodies. Med Hypotheses 2008; 71: 218-221.

34. Najjar S, Pearlman D, Devinsky O, et al. Neuropsychiatric autoimmune encephalitis without VGKC-complex, NMDAR, and GAD autoantibodies: case report and literature review. Cogn Behav Neuro 2013; 26: 36-49.

35. Gresa-Arribas N, Ariño H, Martínez-Hernández E, et al. Antibodies to inhibitory synaptic proteins in neurological syndromes associated with glutamic acid decarboxylase autoimmunity. PLoS One. 2015; 10: $\mathrm{e} 0121364$.

36. Rout UK, Mungan NK, Dhossche DM. Presence of GAD65 autoantibodies in the serum of children with autism or ADHD. Eur ChildAdolesc Psychiatry 2012; 21: 141-147.

37. Jarius S, Stich O, Speck J, et al. Qualitative and quantitative evidence of anti-glutamic acid decarboxylase-specific intrathecal antibody synthesis in patients with stiff person syndrome. J Neuroimmunol 2010; 229: 219-224.

38. Fatemi SH. Cerebellar pathology in autism. In: Gruol DL, Koibuchi N, Manto M, Molinari M, Schmahmann JD, Shen Y (eds). Essentials of Cerebellum and Cerebellar Disorders. Switzerland: Springer, 2016: 539-543.
39. Haberlandt E, Bast $T$, Ebner A, et al. Limbic encephalitis in children and adolescents. Arch Dis Child 2011; 96: 186-191.

40. Kalra S, Burbelo PD, Bayat A, et al. No evidence of antibodies against GAD65 and other specific antigens in children with autism. BBA Clin 2015; 4: 81-84.

41. Bayram AK, Kardas F, Demirci EO, et al. Lack of serum antineuronal antibodies in children with autism. Bratisl Lek Listy 2016; 117: 77-79.

42. Hamed NO, Al-Ayadhi L, Osman MA, et al. Understanding the roles of glutamine synthetase, glutaminase, and glutamate decarboxylase autoantibodies in imbalanced excitatory/inhibitory neurotransmission as etiological mechanisms of autism. Psychiatry Clin Neurosci 2018; 72: 362-373.

43. Stefanatos G. Changing perspectives on LandauKleffner syndrome. Clin Neuropsychol 2011; 25: 963-988.

44. Shenoy S, Arnold S, Chatila T. Response to steroid therapy in autism secondary to autoimmune lymphoproliferative syndrome. J Pediatr 2000; 136: 682-687.

45. Duffy FH, Shankardass A, McAnulty GB, et al. Corticosteroid therapy in regressive autism: a retrospective study of effects on the Frequency Modulated Auditory Evoked Response (FMAER), language, and behavior. BMC Neurol 2014; 14: 70.

46. Malek M, Ashraf-Ganjouei A, Moradi K, Bagheri S, Mohammadi MR, Akhondzadeh S. Prednisolone as adjunctive treatment to risperidone in children with regressive type of autism spectrum disorder: a randomized, placebo-controlled trial. Clin Neuropharmacol 2020; 43: 39-45.

47. Connery K, Tippett M, Delhey LM, et al. Intravenous immunoglobulin for the treatment of autoimmune encephalopathy in children with autism. Trans Psychiatry 2018; 8: 148.

48. Hafizi S, Tabatabaei D, Lai MC. Review of clinical studies targeting inflammatory pathways for individuals with autism. Front Psychiatry 2019; 10: 849 .

49. Ozonoff S, Li D, Deprey L, Hanzel EP, Iosif AM. Reliability of parent recall of symptom onset and timing in autism spectrum disorder. Autism 2018; 22: $891-896$

50. Pearson N, Charman T, Happé F, Bolton PF, McEwen FS. Regression in autism spectrum disorder: reconciling findings from retrospective and prospective research. Autism Res 2018; 11: 16021620. 